\title{
Evaluating Medicare Performance: Perceptions of Performance Pre- and Post- ACA
}

\author{
Ashleigh C. Oguagha ${ }^{1, *} \&$ Nickesha J. Lambert ${ }^{2}$ \\ ${ }^{1}$ School of Public Administration, Huazhong University of Science and Technology, Wuhan, China \\ ${ }^{2}$ Teacher's College, Ball State University, Muncie, United States \\ *Correspondence: School of Public Administration, Huazhong University of Science and Technology, Wuhan, China. \\ E-mail: aoguagha@yahoo.com
}

Received: April 3, $2020 \quad$ Accepted: March 14, $2020 \quad$ Online Published: April 26, 2020

doi:10.5430/wjss.v7n2p28 URL: https://doi.org/10.5430/wjss.v7n2p28

\begin{abstract}
Medicare is a national single-payer system that provides health coverage for the elderly, disabled, and terminally ill in the United States. Rising enrollment, costs, and decreases in financing options may affect the way Medicare performs. This study aimed to investigate participants' perceptions of Medicare performance before and after the Affordable Care Act. A 3-part questionnaire was created and validated for use in this study. Respondents affiliated with several social work agencies were invited via email to participate in this study. 287 out of 519 invited questionnaires were used in data analyses. 27.5\% of respondents reported being currently enrolled in Medicare, while 33.2\% reported lifetime enrollment. Overall, retired/disabled, elderly and low-income participants reported currently or ever using Medicare. One's perception of Medicare performance was determined by their status as a Medicare beneficiary. Medicare service efficacy was rated more positively over-time by current and life-time enrollees; additionally, Medicare performance was determined to be better in 2013 than in 2009. Ultimately, this study showed that health service and financial fairness factors are indicative of Medicare performance. Additional research should explore possible implications for the healthcare field as well as formulate a broad range of possible management and/or improvement strategies. Lastly, differences in performance across years can inform decision-makers and bolster the fundamental foundation of health policies at the state and national level.
\end{abstract}

Keywords: Medicare performance, affordable care act, user perceptions, financial fairness

\section{Introduction}

Medicare is a single-payer system that provides health insurance for American citizens over the age of 65, people with disabilities (regardless of age), and individuals with end-stage renal disease or amyotrophic lateral sclerosis. It was originally controlled by the Social Security Administration, but now it is administered by the Centers for Medicare and Medicaid Services (CMS). Medicare is supposed to provide coverage for eligible citizens, however there is a wide variation in health outcomes for elderly individuals across different states, even if they have similar levels of income and education (U.S. Bureau of the Census, 2018). Since its inception, the Medicare program has been diminishing rapidly, from an annual average growth of 17.2\% in 1980 to 5\% in 2010 (Martin et al., 2012). Its growth rate may be diminishing however the number of Medicare enrollees and expenditures are increasing yearly. From 2000 to 2010 alone, the number of Medicare beneficiaries and hospital days increased by $20.3 \%$ and $4 \%$, respectively (Halpem, Goldman, Tan, \& Pastores, 2016). Because the number of people being covered by Medicare is increasing rapidly, Medicare has undergone financial reforms in order to continue its promise to provide healthcare coverage for the elderly and disabled. Most recently the Affordable Care Act (ACA) has significantly impacted beneficiaries' ability to receive care. Differences in the construction and management of Medicare across state lines can translate into differences in health providers' responsiveness and fairness as well as individual health outcomes.

Medicare reform agenda revolves around themes such as healthcare cost, quality of care and efficiency. Medicare is supposed to serve our most vulnerable however one should question if it is meeting its intended purpose. Jencks et al. (2003) believed that absolute improvement occurs when there is a change in performance between baseline and 
follow-up whereas relative improvement is absolute improvement divided by the variation between baseline and perfect performance $(100 \%)$. Ultimately, performance measures are vital for CMS to effectively meet the public assistance needs outlined in national legislation. Considering its importance to society and social progress, Medicare programs should be continuously monitored and modified according to societal needs. Moreover, comparable information on Medicare performance and factors that explain its performance differences can inform decision-makers and bolster the fundamental foundation of health policies at state and national levels. Therefore, the purpose of this investigation is to explore occupational views of Medicare performance over time.

\section{Methods}

An online survey was used to assess the perceptions and attitudes of various occupations towards Medicare health services and financial fairness. A validated version of the questionnaire was hosted by Surveymonkey.com and required an estimated 5 minutes to complete. It consisted of a study description page, and the following three sections: Section A, Section B, and Section C. In Section A participants completed questions related to their personal and work demographics, Section B consisted of participant's perceptions of Medicare health services and Section C consisted of participant's perceptions of Medicare financial fairness. Sections B and C altogether employed 40 Likert-scale items; 20 of which focused on the year 2009 and the remaining 20 focused on the year 2013.

Study participants consisted of individuals residing in 1 of the continental US 50 states. All participants anonymously, and freely completed the survey. The survey was distributed by administrating agencies such as the Network for Social Work Management, Clinical Social Work Association, Society for Social Work and Research (SSWR), Association of Social Work Boards (ASWB), and National Association of Social Workers (NASW). All those who received the study recruitment email were affiliated with the aforementioned agencies and/or an enrollee in their approved mailing lists. The Ethics Committee of Huazhong University of Science and Technology approved this research. Prior IRB approval was gained from the above agencies before they distributed the survey on behalf of the researchers. The survey was open from July 2019 until October 2019.

\subsection{Indicators of Medicare Performance}

In this study, Medicare performance will be evaluated using health improvement outcomes and financial fairness. Health improvement outcomes for Medicare will be measured using factors such as inpatient and outpatient days (Zuckerman et al., 2016), as well as chronic condition prevention and treatment for individuals 65 years old or older (Thorpe, Ogden, \& Galactionova, 2010). For the purpose of this investigation, chronic conditions can be defined as "conditions that last 1 year or more and require ongoing medical attention or limit activities of daily living or both" (Center for Disease Control [CDC], 2019: https://www.cdc.gov/chronicdisease/about/index.htm). The leading types of chronic conditions are heart disease, cancer, chronic obstructive pulmonary disease (COPD), stroke, Alzheimer's disease, diabetes, and chronic kidney disease (CDC, 2019; National Health Council, 2014).

Financial fairness is the second measure of Medicare performance. There is no set indicator of health improvement in the United States nor is there a framework to measure performance (Braithwaite et al. 2017) however there are numerous national and regional quality improvement initiatives (Kessell et al. 2015),, all of which have a financial incentive tied to their application. Moreover, the amount of financing that is behind the Medicare program will determine how responsive it is to an individual's health needs. Financial fairness can be captured using expenses such as contribution to Medicare through taxes (Safran et al., 2002), total expenditures, and Medicare payouts (Thorpe et al., 2010) as well as median income.

\subsection{Data Analysis}

Statistical analyses were performed using SPSS version 25.0 and SmartPLS 3.2. The reliability and validity of the questionnaire were determined using SmartPLS. Assessment of the validity and reliability were performed using the coefficient of Cronbach's alpha, composite reliability (CR) and average variance expected (AVE). Table 1 depicts the results of a reliability analysis carried out on the perceived Medicare performance efficacy scale. Cronbach's alpha showed the questionnaire constructs to reach acceptable reliability of $\alpha>0.80$. CR and AVE were determined using step analysis in SmartPLS. A minimum Cronbach's alpha (Henseler, 2017) and CR value of 0.70 or higher indicates that the instrument in question is reliable. Items with a Cronbach's alpha of less than 0.40 were dropped due to low corrected-item correlation. Constructs with an AVE of less than 0.5 are still convergently valid if the construct's outer model met the minimum threshold of acceptable reliability and validity (Henseler, 2017). 
Table 1. Questionnaire Reliability and Validity

\begin{tabular}{lllllll}
\hline Construct & Original items & Remaining items & Alpha Value* & Mean & CR & AVE \\
\hline $\begin{array}{l}\text { Health Improvement } \\
\text { Outcomes }\end{array}$ & 20 & 20 & 0.873 & 3.26 & 0.811 & 0.355 \\
Financial Fairness & 20 & 20 & 0.766 & 3.11 & 0.897 & 0.189 \\
Total & 40 & 40 & & & \\
\hline
\end{tabular}

\section{*0.70 or higher}

Descriptive statistical methods were used to calculate participant personal and occupational demographics. Chi-squared analyses were used to explore the relationship between demographic characteristics and Medicare beneficiary status. Univariate analysis such as paired samples t-tests were implemented in order to draw inferences about sample means and variances. Lastly, mixed-Model ANOVAs were used to explore change in perceptions over time using participant characteristics. Statistical significance for all statistical tests was determined using a $\mathrm{P}$ value of less than 0.05 .

\section{Results}

A total of 303 respondents out of 519 delivered questionnaires decided to respond to the survey invitation. Only 287 respondents agreed to continue with the study, for an effective response rate of $55.3 \%$. The participants' demographic and professional characteristics are described in Table 2. The majority of respondents were from the South, reported a household income of between $\$ 25,000$ and $\$ 49,999$, and identified ethnically as White/Caucasian. Nearly an equal number of males and females participated in this study with only $4.2 \%$ preferring not to answer gender-related questions. Age wise, $38.5 \%, 35.7 \%$ and $25.8 \%$ of respondents were respectively considered to be young adults (ages 18-34, $\mathrm{n}=109$ ), middle-aged adults (ages 35-54, $\mathrm{n}=101$ ) and older adults (aged 55+, $\mathrm{n}=73$ ). Occupationally, over $10 \%$ of participants worked in management occupations as well as healthcare and social assistance occupations. $10 \%$ of participants reported that they were retired and/or disabled and $9 \%$ reported that they were unemployed. Furthermore, over $60 \%$ of the respondents reported that they have less than 10 years' experience in their occupational field $(n=181)$. Regarding Medicare enrollment, the majority of participants reported that they are not currently a Medicare recipient, and nor have they ever been a recipient.

Table 2. Respondents’ Demographic Characteristics

\begin{tabular}{lcc}
\hline Characteristics & No & \% \\
\hline Gender & $156 / 287$ & 54.4 \\
Female & $119 / 287$ & 41.5 \\
Male & $12 / 287$ & 4.2 \\
Prefer not to answer/unknown & & 75.3 \\
Ethnicity & $216 / 287$ & 9.4 \\
White/Caucasian & $27 / 287$ & 8.4 \\
Black/African American & $24 / 287$ & 6.9 \\
Asian/pacific islander & $20 / 287$ & 4.6 \\
Other & & 12.4 \\
Age groups & $13 / 283$ & 9.9 \\
$<\mathbf{1 9}$ years old & $35 / 283$ & 11.7 \\
$\mathbf{2 0 - 2 4}$ years old & $28 / 283$ & 6.4 \\
$\mathbf{2 5 - 2 9}$ years old & $33 / 283$ & 6.7 \\
$\mathbf{3 0 - 3 4}$ years old & $18 / 283$ & 12.7 \\
$\mathbf{3 5 - 3 9}$ years old & $19 / 283$ & 9.9 \\
40-44 years old & $36 / 283$ & 8.8 \\
$\mathbf{4 5 - 4 9}$ years old & $28 / 283$ & 17 \\
$\mathbf{5 0 - 5 4}$ years old & $25 / 283$ & \\
$\mathbf{5 5 - 5 9}$ years old & $48 / 283$ & \\
$\mathbf{6 0 +}$ years old & & \\
\hline
\end{tabular}




\begin{tabular}{|c|c|c|}
\hline \multicolumn{3}{|l|}{ Region } \\
\hline South & $96 / 286$ & 33.6 \\
\hline West & $72 / 286$ & 25.2 \\
\hline Midwest & $56 / 286$ & 19.6 \\
\hline Northeast & $62 / 286$ & 21.7 \\
\hline \multicolumn{3}{|l|}{ Household Income } \\
\hline \$0-\$9,999 & $23 / 254$ & 9.1 \\
\hline$\$ 10,000-\$ 24,999$ & $33 / 254$ & 13.0 \\
\hline$\$ 25,000-\$ 49,999$ & $55 / 254$ & 21.7 \\
\hline$\$ 50,000-74,999$ & $49 / 254$ & 19.3 \\
\hline$\$ 75,000-\$ 99,999$ & $40 / 254$ & 15.7 \\
\hline$\$ 1000,000-\$ 124,999$ & $22 / 254$ & 8.7 \\
\hline$\$ 125,000+$ & $32 / 254$ & 12.6 \\
\hline \multicolumn{3}{|l|}{ Job Sector ${ }^{\#}$} \\
\hline Art/Design/Entertainment/Sports/Media & $9 / 279$ & 3.2 \\
\hline Building/Grounds Cleaning \& Maintenance & $9 / 279$ & 3.2 \\
\hline Business Operations Specialists $\dagger$ & $20 / 279$ & 7.2 \\
\hline Computer/Mathematical Occupations & $16 / 279$ & 5.7 \\
\hline Construction and Extraction Occupations & $9 / 279$ & 3.2 \\
\hline Educational Instruction \& Library Occupations & $20 / 279$ & 7.2 \\
\hline Healthcare and Social Assistance Occupations & $30 / 279$ & 10.8 \\
\hline Management Occupations & $29 / 279$ & 10.4 \\
\hline Office and Administrative Support Occupations & $11 / 279$ & 3.9 \\
\hline Personal Care and Service Occupations & $13 / 279$ & 4.7 \\
\hline Protective Service Occupations & $7 / 279$ & 2.5 \\
\hline Sales and Related Occupations & $21 / 279$ & 7.5 \\
\hline Student & $21 / 279$ & 7.5 \\
\hline Retired/Disabled & $29 / 279$ & 10.4 \\
\hline Unemployed & $25 / 279$ & 9.0 \\
\hline Other & $10 / 279$ & 3.6 \\
\hline \multicolumn{3}{|l|}{ Years of occupational experience } \\
\hline$<1$ year & $36 / 286$ & 12.6 \\
\hline $1-5$ years & $87 / 286$ & 30.4 \\
\hline 6-10 years & $58 / 286$ & 20.3 \\
\hline 11-15 years & $21 / 286$ & 7.3 \\
\hline $16-20$ years & $15 / 286$ & 5.2 \\
\hline $21+$ years & $69 / 289$ & 24.1 \\
\hline \multicolumn{3}{|l|}{ Current Medicare Recipient } \\
\hline Yes & $77 / 280$ & 27.5 \\
\hline No & $203 / 280$ & 72.5 \\
\hline \multicolumn{3}{|l|}{ Former Medicare recipient } \\
\hline Yes & $93 / 280$ & 33.2 \\
\hline No & $187 / 280$ & 66.8 \\
\hline
\end{tabular}

$\dagger$ Includes Financial Specialists

\#available at https://www.bls.gov/soc/2018/major_groups.htm\#27-0000

\subsection{Medicare Enrollment}

Over a quarter of participants reported that they are currently receiving Medicare and/or have ever received Medicare (Table 2). The majority of participants who reported currently or ever receiving Medicare identified as White/ Caucasian (77\%; 75.0\%), and older than $60(37.7 \%$; 30.4\%). More male responses reported current enrollment in 
Medicare (52.8\%) whereas females had higher frequencies of lifetime enrollment (51.7\%). The majority of current and lifetime beneficiaries reported living in the South and Northeast. Financially, $25.6 \%$ and $23.9 \%$ of lifetime and current enrollees reported an annual household income of between $\$ 25,000$ and $\$ 49,999$. For both lifetime and current enrollees most reported being currently employed however $31.2 \%$ of lifetime enrollees reported have between 1- and 5-years' current work experience whereas 31.2\% of current enrollees reported having more than 20+ years' experience at their job. Of those considered to be unemployed (i.e., participants identifying as disabled/unable to work, retired, unemployed, and students), $59 \%$ and $67.6 \%$ of lifetime and current Medicare enrollees respectively reported being disabled/retired.

Using Chi-square analysis, a significant difference was among age, household income, and job industry (Table 3) according to enrollment status. Results showed that young adults $(\mathrm{n}=37,40.2 \%)$ compared to older adults $(\mathrm{n}=32$, $34.8 \%)$ and middle-aged adults $(\mathrm{n}=23,25 \%)$ had higher frequencies of lifetime Medicare enrollment. Participants with a household income level of less than $\$ 50,000(n=42,59.8 \%)$ had higher lifetime Medicare enrollment compared to those with a higher income bracket. Occupationally, employed participants had higher lifetime enrollment compared to unemployed participants, $\chi 2(1, \mathrm{~N}=274)=17.491, \mathrm{p}=00$. More specifically, management occupations, educational instruction and library occupations, as well as business operations specialists, had higher frequencies of Medicare enrollment compared to other industries (Table 3). Meanwhile, analyses showed that older adults $(n=33,42.9 \%)$ compared to young adults $(n=26,33.8 \%)$ and middle-aged adults $(n=18,23.4 \%)$ had higher frequencies of current Medicare enrollment. Participants with a household income level of less than $\$ 50,000(n=39$, $58.2 \%$ ) had higher Medicare enrollment compared to those with a higher income bracket. Occupationally, a significant number of unemployed participants are currently receiving Medicare, $\chi^{2}(1, \mathrm{~N}=274)=16.336, \mathrm{p}=00$. More specifically, retired/disabled workers had higher frequencies of Medicare enrollment compared to other unemployed and employed participants (Table 3).

Table 3. Chi-square Analysis of Participant Medicare Enrollment

\begin{tabular}{|c|c|c|c|c|c|c|}
\hline \multirow[b]{2}{*}{ Characteristics } & \multicolumn{3}{|c|}{ Lifetime Enrollees } & \multicolumn{3}{|c|}{ Current Enrollees } \\
\hline & No $(\%)$ & $\chi 2$ (df) & $\mathbf{P}$ & No (\%) & $\chi 2$ (df) & $\mathbf{P}$ \\
\hline Gender & & $0.705(1)$ & 0.401 & & $2.591(1)$ & 0.107 \\
\hline Female & $45(30)$ & & & $34(22.7)$ & & \\
\hline Male & $42(35.6)$ & & & $38(32)$. & & \\
\hline Ethnicity & & $4.787(3)$ & 0.188 & & $0.777(3)$ & 0.855 \\
\hline White/Caucasian & $66(31.0)$ & & & $57(26.8)$ & & \\
\hline Black/African American & $12(52.2)$ & & & $8(34.8)$ & & \\
\hline Asian/pacific islander & $8(33.3)$ & & & $6(25)$ & & \\
\hline Other & $2(20)$ & & & $3(30)$ & & \\
\hline Age groups & & $29.782(9)$ & $0.000 * *$ & & $33.691(9)$ & $0.000 * *$ \\
\hline$<19$ years old & $6(46.2)$ & & & $5(38.5)$ & & \\
\hline $20-24$ years old & $16(45.7)$ & & & $8(22.9)$ & & \\
\hline 25-29 years old & $7(25.9)$ & & & $6(22.2)$ & & \\
\hline $30-34$ years old & $8(24.2)$ & & & $7(21.2)$ & & \\
\hline 35-39 years old & $4(22.2)$ & & & $3(16.7)$ & & \\
\hline 40-44 years old & $7(36.8)$ & & & $4(21.1)$ & & \\
\hline 45-49 years old & $4(11.4)$ & & & $5(14.3)$ & & \\
\hline $50-54$ years old & $8(30.8)$ & & & $6(23.1)$ & & \\
\hline 55-59 years old & $4(18.2)$ & & & $4(18.2)$ & & \\
\hline $60+$ years old & $28(58.3)$ & & & $29(60.4)$ & & \\
\hline Region & & $4.209(3)$ & 0.240 & & $5.246(3)$ & 0.155 \\
\hline South & $30(32.3)$ & & & $24(25.8)$ & & \\
\hline West & $24(33.8)$ & & & $17(23.9)$ & & \\
\hline Midwest & $13(24.1)$ & & & $12(22.2)$ & & \\
\hline Northeast & $26(41.9)$ & & & $24(38.7)$ & & \\
\hline
\end{tabular}




\begin{tabular}{|c|c|c|c|c|c|c|}
\hline Household Income & & $17.253(6)$ & $0.008^{* *}$ & & $13.640(6)$ & $0.34 *$ \\
\hline \$0-\$9,999 & $12(52.2)$ & & & $10(43.5)$ & & \\
\hline$\$ 10,000-\$ 24,999$ & $16(48.5)$ & & & $13(39.4)$ & & \\
\hline$\$ 25,000-\$ 49,999$ & $21(38.2)$ & & & $16(29.1)$ & & \\
\hline$\$ 50,000-74,999$ & $11(22.4)$ & & & $9(18.4)$ & & \\
\hline$\$ 75,000-\$ 99,999$ & $11(27.5)$ & & & $10(25)$ & & \\
\hline$\$ 1000,000-\$ 124,999$ & $2(9.1)$ & & & $1(4.5)$ & & \\
\hline$\$ 125,000+$ & $9(28.1)$ & & & $8(25)$ & & \\
\hline Job Sector $\#$ & & $39.845(15)$ & $0.000 * *$ & & $48.820(15)$ & $0.000 * *$ \\
\hline $\begin{array}{l}\text { Art/Design/Entertainment/ } \\
\text { Sports/Media }\end{array}$ & $1(11.1)$ & & & $1(11.1)$ & & \\
\hline $\begin{array}{l}\text { Building/Grounds Cleaning } \\
\text { \& Maintenance }\end{array}$ & $1(11.1)$ & & & $1(11.1)$ & & \\
\hline $\begin{array}{l}\text { Business Operations } \\
\text { Specialists } \dagger\end{array}$ & $6(30)$ & & & $6(30)$ & & \\
\hline $\begin{array}{l}\text { Computer/Mathematical } \\
\text { Occupations }\end{array}$ & $5(31.3)$ & & & $5(31.3)$ & & \\
\hline $\begin{array}{l}\text { Construction and } \\
\text { Extraction Occupations }\end{array}$ & $3(33.3)$ & & & $2(22.2)$ & & \\
\hline $\begin{array}{l}\text { Educational Instruction \& } \\
\text { Library Occupations }\end{array}$ & $6(30)$ & & & $5(25)$ & & \\
\hline $\begin{array}{l}\text { Healthcare and Social } \\
\text { Assistance Occupations }\end{array}$ & $5(20)$ & & & $5(20)$ & & \\
\hline Management Occupations & $6(20.7)$ & & & $6(20.7)$ & & \\
\hline $\begin{array}{l}\text { Office and Administrative } \\
\text { Support Occupations }\end{array}$ & $4(36.4)$ & & & $2(18.2)$ & & \\
\hline $\begin{array}{l}\text { Personal Care and Service } \\
\text { Occupations }\end{array}$ & $2(15.4)$ & & & $3(23.1)$ & & \\
\hline $\begin{array}{l}\text { Protective Service } \\
\text { Occupations }\end{array}$ & $1(14.3)$ & & & - & & \\
\hline $\begin{array}{l}\text { Sales and Related } \\
\text { Occupations }\end{array}$ & $5(23.8)$ & & & $2(9.5)$ & & \\
\hline Student & $7(33.3)$ & & & $4(19)$ & & \\
\hline Retired/Disabled & $23(79.3)$ & & & $23(79.3)$ & & \\
\hline Unemployed & $9(36)$ & & & $7(28)$ & & \\
\hline Other & $4(40)$ & & & $2(20)$ & & \\
\hline Occupational experience & & $6.514(5)$ & 0.259 & & $6.285(5)$ & 0.279 \\
\hline$<1$ year & $15(41.7)$ & & & $10(27.8)$ & & \\
\hline $1-5$ years & $29(33.7)$ & & & $23(26.7)$ & & \\
\hline 6-10 years & $16(28.1)$ & & & $14(24.6)$ & & \\
\hline $11-15$ years & $3(14.3)$ & & & $2(9.5)$ & & \\
\hline $16-20$ years & $4(28.6)$ & & & $4(28.6)$ & & \\
\hline $20+$ years & $26(39.4)$ & & & $24(36.4)$ & & \\
\hline
\end{tabular}

$\dagger$ Includes Financial Specialists

\#available at https://www.bls.gov/soc/2018/major_groups.htm\#27-0000

$* \mathbf{p}<.05, * * \mathbf{p}<.01$ 


\subsection{Perceptions of Medicare Over Time}

\subsubsection{Perceptions of Medicare Health Services}

One of the main issues investigated in this study was respondents' perceptions of Medicare health improvement before and after 2010. Respondents were asked to rate Medicare health service efficacy on a Likert scale of 1 (Strongly Disagree) to 5 (Strongly Agree). A paired sample t-test found that there was a significance different between people's perception of Medicare's 2009 and 2013 performance, $t(277)=-4.63, p<0.001$. Subsequently, a repeated-measures ANOVA with participant characteristics as the between-subjects factor and health service perceptions over time as a within-subjects factor was executed. Analysis revealed that a significant difference within the main effect of Medicare health service efficacy perceptions, $\mathrm{p}<0.05$, however, no significance was found between-subjects for gender, age, ethnicity, region, household income, job sector, employment status, length of employment and Medicare lifetime enrollment nor for interactions between service efficacy perceptions and the aforementioned variables (Table 4).

A repeated-measures ANOVA using Greenhouse-Geisser corrections found that participant perceptions of Medicare health service efficacy over time depends on their current Medicare enrollment status. Participants who were currently in Medicare rated Medicare service efficacy more positively over time compared to those who were not currently enrolled in Medicare. For current and non-Medicare enrollees Medicare performance was perceived to be better in 2013 compared to 2009.

Table 4. Mixed Model ANOVA for Service Efficacy and Participant Characteristics

\begin{tabular}{|c|c|c|c|c|c|}
\hline Variable & & & $\mathbf{F}$ & $p$ & Eta squared \\
\hline Service Efficacy & 1 & 265 & 18.905 & 0.000 & 0.067 \\
\hline Service Efficacy ${ }^{*}$ Gender & 1 & 265 & 0.428 & 0.514 & 0.002 \\
\hline Service Efficacy & 1 & 264 & 20.562 & 0.000 & 0.072 \\
\hline Service Efficacy*Age & 9 & 264 & 0.969 & 0.466 & 0.032 \\
\hline Service Efficacy & 1 & 264 & 10.085 & 0.002 & 0.037 \\
\hline Service Efficacy*Ethnicity & 3 & 264 & 0.381 & 0.767 & 0.004 \\
\hline Service Efficacy & 1 & 273 & 23.317 & 0.000 & 0.079 \\
\hline Service Efficacy*Region & 3 & 273 & 0.790 & 0.500 & 0.009 \\
\hline Service Efficacy & 1 & 247 & 20.862 & 0.000 & 0.078 \\
\hline Service Efficacy*Income & 6 & 247 & 1.615 & 0.144 & 0.038 \\
\hline Service Efficacy & 1 & 255 & 17.337 & 0.000 & 0.064 \\
\hline Service Efficacy*Job Sector & 15 & 255 & 0.897 & 0.568 & 0.050 \\
\hline Service Efficacy & 1 & 269 & 12.821 & 0.000 & 0.045 \\
\hline $\begin{array}{l}\text { Service Efficacy*Employment } \\
\text { Status }\end{array}$ & 1 & 269 & 0.906 & 0.342 & 0.003 \\
\hline Service Efficacy & 1 & 271 & 13.777 & 0.000 & 0.048 \\
\hline $\begin{array}{l}\text { Service Efficacy*Length of } \\
\text { Employment }\end{array}$ & 5 & 271 & 0.288 & 0.920 & 0.005 \\
\hline Service Efficacy $\dagger$ & 1 & 272 & 13.053 & 0.000 & 0.046 \\
\hline $\begin{array}{l}\text { Service Efficacy*Current } \\
\text { Medicare enrollment }\end{array}$ & 1 & 272 & 5.648 & 0.018 & 0.020 \\
\hline Service Efficacy $\dagger$ & 1 & 275 & 13.917 & 0.000 & 0.049 \\
\hline $\begin{array}{l}\text { Service Efficacy*Lifetime } \\
\text { Medicare Enrollment }\end{array}$ & 1 & 275 & 1.734 & 0.189 & 0.006 \\
\hline
\end{tabular}

$\dagger$ Uses Greenhouse-Geisser due to violation of Levene's test of Equality 


\subsection{Perceptions of Medicare Financial Fairness}

Another issue investigated in this study was respondents' perceptions of Medicare financial fairness before and after 2010. Respondents were asked to rate Medicare financial fairness on a Likert scale of 1 (Strongly Disagree) to 5 (Strongly Agree). A paired sample t-test found no significant difference between people's perception of Medicare's financial fairness from 2009 to 2013, $\mathrm{t}(271)=1.01, \mathrm{p}=0.315$. A repeated-measures ANOVA with participant characteristics as the between-subjects factor and perceptions over time as a within-subjects factor was run. Analysis revealed that no significant difference within the main effect of Medicare Financial Fairness perceptions, was found, p > 0.05; additionally, no significance was found between-subjects for gender, age, ethnicity, region, household income, job sector, employment status, and length of employment nor for interactions between financial fairness perceptions and the aforementioned variables (Table 5).

However, a repeated-measures ANOVA using a Greenhouse-Geisser correction found that participant perceptions of Medicare financial fairness over time depends on their current and lifetime Medicare enrollment status. Participants who were currently or ever enrolled in Medicare held more positive perceptions of Medicare financial fairness over time compared to those who were not currently enrolled in Medicare. For current and lifetime Medicare enrollees, Medicare financial fairness was perceived to be better in 2013 compared to 2009 however for those not enrolled in Medicare now or ever they perceived Medicare to be more financially fair in 2009 as opposed to 2013.

Table 5. Mixed Model ANOVA for Financial Fairness and Participant Characteristics

\begin{tabular}{|c|c|c|c|c|c|}
\hline \multirow{2}{*}{$\begin{array}{r}\text { Variable } \\
\text { Financial Fairness }\end{array}$} & \multicolumn{2}{|c|}{ df } & \multirow{2}{*}{$\begin{array}{c}\mathbf{F} \\
1.159\end{array}$} & \multirow{2}{*}{$\begin{array}{c}p \\
0.283\end{array}$} & \multirow{2}{*}{$\begin{array}{c}\text { Eta squared } \\
0.004\end{array}$} \\
\hline & 1 & 259 & & & \\
\hline Financial Fairness*Gender & 1 & 259 & 1.319 & 0.252 & 0.005 \\
\hline Financial Fairness & 1 & 258 & 0.617 & 0.433 & 0.002 \\
\hline Financial Fairness*Age & 9 & 258 & 1.635 & 0.105 & 0.054 \\
\hline Financial Fairness & 1 & 258 & 0.678 & 0.411 & 0.003 \\
\hline Financial Fairness*Ethnicity & 3 & 258 & 0.305 & 0.822 & 0.004 \\
\hline Financial Fairness & 1 & 278 & 0.985 & 0.322 & 0.004 \\
\hline Financial Fairness*Region & 3 & 278 & 0.719 & 0.541 & 0.008 \\
\hline Financial Fairness & 1 & 247 & 0.450 & 0.503 & 0.002 \\
\hline Financial Fairness*Income & 6 & 247 & 0.846 & 0.535 & 0.020 \\
\hline Financial Fairness & 1 & 249 & 1.969 & 0.162 & 0.008 \\
\hline Financial Fairness*Job Sector & 15 & 249 & 0.418 & 0.973 & 0.025 \\
\hline Financial Fairness & 1 & 263 & 0.348 & 0.556 & 0.001 \\
\hline $\begin{array}{l}\text { Financial } \\
\text { Fairness*Employment Status }\end{array}$ & 1 & 263 & 0.109 & 0.741 & 0.000 \\
\hline Financial Fairness & 1 & 265 & 0.839 & 0.360 & 0.003 \\
\hline $\begin{array}{l}\text { Financial Fairness* Length of } \\
\text { Employment } \dagger\end{array}$ & 5 & 265 & 1.255 & 0.284 & 0.023 \\
\hline Financial Fairness $\dagger$ & 1 & 266 & 0.001 & 0.973 & 0.000 \\
\hline $\begin{array}{l}\text { Financial Fairness*Current } \\
\text { Medicare enrollment }\end{array}$ & 1 & 266 & 11.226 & 0.001 & 0.40 \\
\hline Financial Fairness $\dagger$ & 1 & 266 & 0.120 & 0.730 & 0.000 \\
\hline $\begin{array}{l}\text { Financial Fairness*Lifetime } \\
\text { Medicare Enrollment }\end{array}$ & 1 & 266 & 6.761 & 0.010 & 0.025 \\
\hline
\end{tabular}

$\dagger$ Uses Greenhouse-Geisser due to violation of Levene's Test of Equality 


\section{Discussion}

This study aimed to explore user and non-user perceptions of Medicare performance through health service outcomes and financial indicators. Results indicated that nearly a third of respondents were currently enrolled in Medicare or ever enrolled in Medicare. This rate is higher than estimates provided by the US government (CMS Office of the Actuary, 2018). Higher incidences of Medicare enrollees could be the result of the willingness of those individuals to participate in this study. However, other participant demographics (i.e., gender, ethnicity, and region) are similar to estimates provided by the U.S. Bureau of the Census (2018).

Overall, both current Medicare enrollment and lifetime enrollment significantly differed according to age, household income, and job industry. Participants who were 60+ years old had lower household income brackets and occupationally retired/disabled individuals had a higher incidence of Medicare enrollment. Age and occupational status differences are the cause of Medicare enrollment requirements because Medicare specifically allocates funds for elderly, retired and/or disabled individuals (Medicare.gov, n.d.). The higher incidence of Medicare enrollees for lower-income brackets is due to Medicare demographics. According to Jacobson, Neuman, and Musumeci (2017), $20 \%$ of the population utilizes Medicare of which 70 million are low-income Americans. Additionally, age and disability requirements set forth by CMS may dissuade healthy, younger Americans from responding to Medicare-related questionnaires.

Data shows that there was a significant difference among user perception of Medicare health services however there were no significant differences among user perception of Medicare financial fairness. Users who were currently enrolled or ever enrolled in Medicare rated Medicare health services as better in 2013 compared to 2009. User perceptions of Medicare service were based on access to care, services for chronic illnesses, and patient priority among other things. More positive attitudes towards health services in 2013 could be the work of ACA and its mandate to expand care for those with preexisting conditions. Although there was no significance between users' perceptions of financial fairness, participants rated Medicare financial fairness more negatively in 2013. The discrepancies between health services and financial fairness could be the result of the subjective nature of the study. Self-completed questionnaires are notorious for low response rates as well as the exclusion of differing opinions (Phellas, Bloch, \& Seale, 2011). Additionally, financial fairness is dependent upon subjective factors such as disposable income and healthcare costs. Previous investigations into America's attitudes post-ACA found that citizens did not like the fact that ACA mandates enrollment in healthcare (Dalen et al., 2015) and perceivably increased health insurance costs (Gordon et al., 2017). Mandated enrollments mean that people will view Medicare more as a financial burden instead of a way to help alleviate medical expenses.

Overall, Medicare performance was determined through the culmination of health service outcomes and financial fairness factors. When considering Medicare performance, participants and users currently enrolled or ever enrolled in Medicare rated Medicare performance as better pre-ACA as opposite to post-ACA. Users rating Medicare performance as more positive in 2013 is in line with Dalen et al. 's (2015) research, which asserts those who benefit more from ACA have more favorable views towards it. Additionally, media portrayal of ACA could disproportionately affect respondents' views of ACA. According to Fowler et al. (2017), individuals who were not exposed to insurance product ads and local media coverage about the ACA marketplace during its implementation held more negative views about Medicare and the ACA reform. Ultimately, Medicare performance perceptions are essentially influenced by a person's proximity to Medicare as well as societal factors such as media and news coverage.

\section{Conclusion}

Medicare performance can first be determined by evaluating if it met its original purpose. Originally, Medicare sought to help people make informed healthcare decisions and access affordable care while post-ACA Medicare sought to primarily reform the healthcare system (CMS, 2008; Silvers, 2013). It is safe to say that Medicare achieved its goal of providing affordable healthcare to beneficiaries. More recently, premiums for Medicare beneficiaries have increased, which coincidence with increased enrollment and changes to private insurance provisions; both of which achieve post-ACA Medicare's goal of expanding care through the reform of the healthcare system. Secondly, this study quantitively shows that Medicare performance improved on the national level before and after ACA implementation. Participants held positive perceptions of overall Medicare performance and Medicare health services post-2010. However, citizens do not believe that Medicare was better financially after ACA implementation. In light of this information, the government needs to do more to enlighten the publication about the uses of Medicare in conjunction with ACA. Additionally, the government can be more transparent about Medicare utilization costs in 
an effort to improve the public's opinion about Medicare. Ultimately, this study can be used to bolster calls for universal healthcare as well as highlight the benefits of having an informed public.

Various limitations are present in this study. Firstly, this study only focused on one of two types of national insurance plan. Assessment of the Medicaid program, versus the Medicare program, may provide different information related to performance and people's perception of performance. Secondly, participant population demographics may cause the data to skew. The population of this study consisted of English-speaking individuals, individuals associated with the selected organizations or those residing in the continental US. Meaning that those not affiliated with the organization or residing in territories that have access to Medicare might have differing opinions about Medicare performance. Additionally, this study has Medicare enrollment numbers higher than national average however, this is balanced due to normalized numbers for other participant demographics (age, race, and gender). Lastly, this study attempted to evaluate Medicare performance at different points, which may be over-generalized due to time-constraints and memory bias. Further examination of the Medicare performance over-time is warranted. Additional assessment of Medicare performance is needed in comparison to Medicaid as well as on an individual state-level.

\section{References}

Braithwaite, J., Hibbert, P., Blakely, B., Plumb, J., Hannaford, N., Long, J. C., \& Marks, D. (2017). Health system frameworks and performance indicators in eight countries: a comparative international analysis. SAGE open medicine, 1-10. https://doi.org/10.1177/2050312116686516

Centers for Disease Control and Prevention. (2019). Chronic Diseases in America. National Center for Chronic Disease Prevention and Health Promotion. Retrieved February 2020 from https://www.cdc.gov/chronicdisease/resources/infographic/chronic-diseases.htm

Centers for Medicare and Medicaid Services. (2008). Medicare \& You 2009. U.S. Department of Health and Human Services. $\quad$ Retrieved $\quad$ February 2020 from https://q1medicare.com/pics/ContentPics/MedicareAndYou2009_10050.pdf\&sa=U\&ved=2ahUKEwizpMmJnJ PoAhVByoUKHd8bAtYQFjAEegQIARAB\&usg=AOvVaw13fzjWd3GL3zNYzUaXriF2

Dalen, J., Waterbrook, K., \& Alpert, J. (2015). Why do so many Americans oppose the Affordable Care Act?. The American Journal of Medicine, 128(8), 807-810. https://doi.org/10.1016/j.amjmed.2015.01.032

Fowler, E. F., Baum, L. M., Barry, C. L., Niederdeppe, J., \& Gollust, S. E. (2017). Media messages and perceptions of the Affordable Care Act during the early phase of implementation. Journal of Health Politics, Policy and Law, 42(1), 167-195. https://doi.org/10.1215/03616878-3702806

Gordon, P. R., Gray, L., Hollingsworth, A., Shapiro, E. C., \& Dalen, J. E. (2017). Opposition to Obamacare: a closer look. Academic Medicine, 92(9), 1241-1247. https://doi.org/10.1097/ACM.0000000000001725

Halpern, N. A., Goldman, D. A., Tan, K. S., \& Pastores, S. M. (2016). Trends in Critical Care Beds and Use Among Population Groups and Medicare and Medicaid Beneficiaries in the United States: 2000-2010. Critical care medicine, 44(8), 1490-1499. https://doi.org/10.1097/CCM.0000000000001722

Henseler, J. (2017). Partial least squares path modeling. In Leeflang P., Wieringa J., Bijmolt T., Pauwels K. (Eds.), Advanced methods for modeling markets, pp. 361-381. Springer, Cham. https://doi.org/10.1007/978-3-319-53469-5_12

Jacobson, G., Neuman, T., \& Musumeci, M. (2017). What could a Medicaid per capita cap mean for low-income people on Medicare?. Menlo Park, CA: Kaiser Family Foundation. Retrieved January 2020 from https://nationaldisabilitynavigator.org/wp-content/uploads/news-items/KFF_Medicaid-Per-Capita-Cap-and-Low -Income-People-on-Medicare_March-2017.pdf

Jencks, S., Huff., E., \& Cuerdon. (2003). Change in the quality of care delivered to Medicare Beneficiaries, 1998-1999 to 2000-2001. JAMA, 289(3), 305-312. https://doi.org/10.1001/jama.289.3.305

Kessell, E., Pegany, V., Keolanui, B., Fulton, B. D., Scheffler, R. M., \& Shortell, S. M. (2015). Review of Medicare, Medicaid, and commercial quality of care measures: considerations for assessing accountable care organizations. Journal of Health Politics, Policy and Law, 40(4), 761-796. https://doi.org/10.1215/03616878-3150050

Martin, A. B., Lassman, D., Washington, B., Catlin, A., \& National Health Expenditure Accounts Team. (2012). Growth in US health spending remained slow in 2010; health share of gross domestic product was unchanged 
from 2009. Health Affairs, 31(1), 208-219. https://doi.org/10.1377/hlthaff.2011.1135

Medicare.gov. (n.d.). The Official U.S. Government Site for Medicare Family of Sites. Retrieved December 2018, from https://www.medicare.gov/

National Health Council (2014). About Chronic Diseases. Retrieved February 2020 from https://nationalhealthcouncil.org/wp-content/uploads/2019/12/AboutChronicDisease.pdf

Office of the Actuary. (2018, February). CMS Office of the Actuary releases 2017-2026 Projections of National Health Expenditures. Centers for Medicare \& Medicaid Services. Retrieved January 2020 from https://www.cms.gov/newsroom/press-releases/cms-office-actuary-releases-2017-2026-projections-national-hea lth-expenditures

Phellas, C., Bloch, A., \& Seale, C. (2011). Structured methods: Interviews, questionnaires and observation. In: Seale (Eds.), Researching Society and Culture. London: SAGE Publications, 181-205.

Safran, D. G., Wilson, I. B., Rogers, W. H., Montgometery, J. E., \& Chang, H. (2002). Primary care quality in the Medicare program: Comparing the performance of Medicare health maintenance organizations and traditional fee-for-service Medicare. Archives of Internal Medicine, 16(2), 757-765. https://doi.org/10.1001/archinte.162.7.757

Silvers, J. B. (2013). The affordable care act: Objectives and likely results in an imperfect world. Annals of Family Medicine, 11(5), 402-405. https://doi.org/10.1370/afm.1567

Thorpe, K., Ogden, L., \& Galactionova. (2010). Chronic conditions account for rise in Medicare spending from 1987 to 2006. Health Affairs, 29(4), 718-724. https://doi.org/10.1377/hlthaff.2009.0474

U.S. Bureau of the Census. (2018). United States Census Bureau Family of Sites. Retrieved January 2020 from https://www.census.gov

Zuckerman, R., Sheingold, D., Orav, E., Ruther, J., \& Epstein. (2016). Readmissions, observation, and the hospital readmissions reduction program. New England Journal of Medicine, 374(16), 1543-1551. https://doi.org/10.1056/NEJMsa1513024 